\title{
Coexistence of Gastric Cancer and Multiple Small Gastrointestinal Stromal Tumors: Report of a Unique Case and Review of the Literature
}

\author{
Helen J. Trihia \\ Department of Pathology, "Metaxas" Cancer Hospital, Piraeus, Greece
}

\author{
Keywords \\ Gastric · Adenocarcinoma - Synchronous - Gastrointestinal · Stromal tumors
}

\begin{abstract}
Background: In recent years, the synchronous occurrence of tumors of different histotypes arising in the same organ has been reported more frequently in the literature. The simultaneous development of adenocarcinoma and gastrointestinal stromal tumor (GIST) has been documented rarely. Summary: The coexistence of primary gastric adenocarcinoma and GIST has been detected incidentally on gastric mucosa, serosa or occasionally intramurally, at surgery, or gastroscopy for other reasons. We present a case of a 79-year-old male patient who underwent surgery for an advanced gastric carcinoma, where multiple nodules of GIST were incidentally discovered during the work-up of his gastrectomy specimen. Key Message: GISTs range from small "low-risk" tumors to sarcomas. Small GISTs are found incidentally during unrelated surgery or autopsy. Multiple GISTs are extremely rare and usually associated with hereditary diseases. Practical Implications: In any case of gastrointestinal neoplasm, the surgeon and pathologist should be alert to perform a thorough investigation. Our case could provide further awareness and insight into the entity of concurrent tumors.
\end{abstract}

(C) 2018 S. Karger AG, Basel

\section{Introduction}

Gastrointestinal stromal tumors (GISTs) are the most common type of mesenchymal tumors in the gastrointestinal (GI) tract, although they comprise $<1 \%$ of all GI tumors. GISTs reportedly can occur synchronously with other GI neoplasms, and according to a case series reported in the literature, the incidence of synchrony between GISTs and gastric adenocar- 
cinoma is $0.53 \%$. We report a case of a 79 -year-old man who presented with gastrorrhagia. Endoscopy revealed a large mass along the lesser curvature. Biopsy was diagnostic of adenocarcinoma of the stomach. Total gastrectomy was performed. The patient succumbed a few days later because of renal failure.

\section{Case Report}

A 79-year-old male was diagnosed with a proximal gastric adenocarcinoma and underwent a total gastrectomy. Subsequent histopathology revealed the presence of an ulcerated mass along the lesser curvature, measuring approximately $8.5 \times 6.5 \times 1 \mathrm{~cm}$. Histological examination revealed a moderately to poorly differentiated, intestinal type, gastric adenocarcinoma. Interestingly, 3 firm neighboring nodules of $0.3,0.5$, and $0.7 \mathrm{~cm}$ were incidentally identified in the subserosa of the gastric wall (Fig. 1), while searching for lymph nodes in the greater curvature. These nodules were composed of cytologically bland spindle cells that were demonstrated immunohistochemically to be uniformly positive for CD117 (c-Kit), actin and CD34 and focally positive for S-100, comprising therefore, small, low-risk GISTs. A fourth microscopic focus of 0.9 mm (Fig. 2) was found in random sections.

\section{Discussion}

GISTs are rare tumors with an estimated incidence of 1.5/100,000/year. This only covers the clinically relevant GISTs, since it is likely that a much higher number of microscopic lesions could be found pathologically, if looked for [1]. GISTs are rare mesenchymal neoplasms of the digestive tract. Synchronous occurrence of a GIST with a tumor of different histogenesis is very rare and has been documented in the literature mainly in case reports. GISTs have been reported to occur synchronously with colon adenocarcinoma, gastric cancer, lymphoma and carcinoid [2-4]. Coexistence with neoplasms in other organs has also been reported, such as pancreatic adenocarcinoma, uterine sarcoma, and thyroid papillary carcinoma [5].

Although the coexistence of GISTs with other neoplasms is rare, it is more common than was previously considered. Liszka et al. [6] estimated that $11.5 \%$ of gastric GISTs occur synchronously with other gastric malignancies, while reports of other series give the perspective percentage of 14,27 , and $33.3 \%$ respectively. The overall frequency of second tumors in different series varies from 4.5 to $33 \%$ (mean 13\%). The major types of GIST-associated cancers are GI carcinomas (47\%), lymphoma/leukemia (7\%), carcinomas of prostate (9\%), breast (7\%), kidney (6\%), lung (5\%), female genital tract (5\%), and carcinoid tumors (3\%). As well as soft tissue and bone sarcomas (3\%), malignant melanoma (2\%) and seminoma (1\%) [7]. GISTs are specific, generally Kit (CD117)-positive, mesenchymal tumors of the GI tract encompassing a majority of tumors previously considered GI smooth muscle tumors. They are believed to originate from interstitial cells of Cajal or related multipotential (pacemaker) cells. GISTs usually occur in older adults (mean 55-60 years) and rarely in children in the second decade $(<1 \%)$ throughout the GI tract: $60 \%$ in the stomach, $35 \%$ in the small intestine and less than $5 \%$ in the rectum, esophagus, omentum, and mesentery. Five percent of GISTs occur in patients with neurofibromatosis type 1 syndrome (multiple small intestinal tumors) and in Carney triad (gastric epithelioid GISTs in young females, paraganglioma and pulmonary chondroma). Familial GISTs occur in patients with inheritable germline Kit or platelet-derived growth factor receptor alpha mutations [8].

Multiple GISTs are extremely rare and usually associated with type 1 neurofibromatosis and familial GIST. In a clinicopathologic and genetic analysis of multiple GISTs from 12 patients, with 5 sporadic, 2 familial, and 5 associated with type 1 neurofibromatosis cases, shared mutations of KIT gene and diffuse involvement of the GI tract by diffuse hyperplasia 


\section{Gastro Intestinal Tumors}

Fig. 1. Nodule of $0.7 \mathrm{~cm}$ was incidentally found in the subserosa of the gastric wall. This nodule was composed of cytologically bland spindle cells that were demonstrated immunohistochemically to be uniformly positive for CD117.

Fig. 2. Nodule of $0.9 \mathrm{~mm}$ was incidentally found in the subserosa of the gastric wall in random sections. This nodule was composed of cytologically bland spindle cells that were demonstrated immunohistochemically to be uniformly positive for CD117.

\begin{tabular}{l|l}
\hline Gastrointest Tumors 2018;5:63-67 \\
\hline DOI: 10.1159/000495178 & $\begin{array}{l}\text { @ 2 2018 S. Karger AG, Basel } \\
\text { www.karger.com/gat }\end{array}$ \\
\hline
\end{tabular}
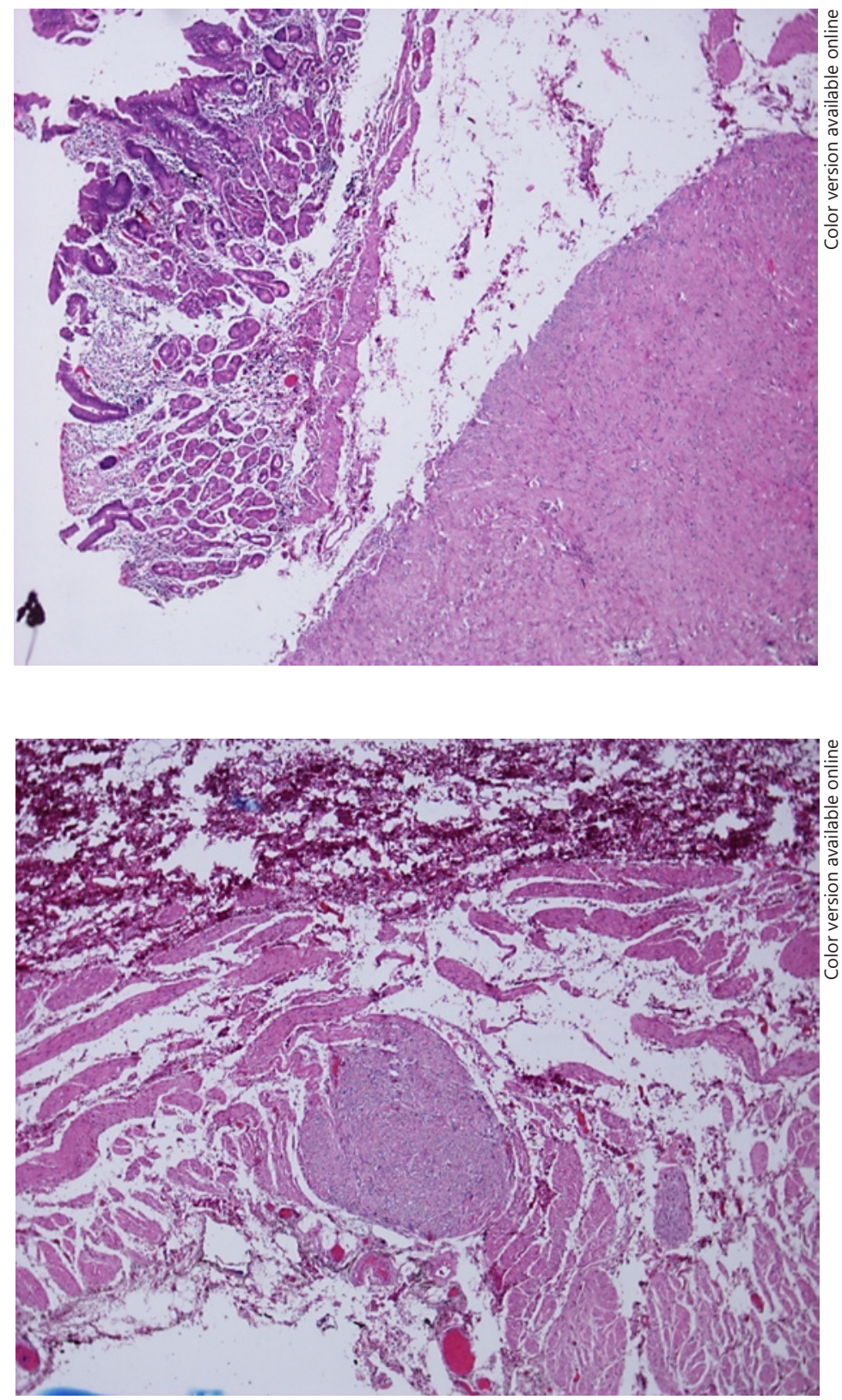

of interstitial cells of Cajal [9]. Multiple GISTs show a favorable prognosis regardless of the number or phenotype of GISTs.

Despite the great progress made in recent years in understanding the molecular biology of GISTs, little is yet known about their rare synchronous occurrence with tumors of different histogenesis. In earlier Japanese literature, a few cases of coexistence of gastric leiomyomatous tumors, with gastric cancer have been reported, but immunohistochemical staining for CD-117 has never been confirmed [10]. Although in most of the reported cases, the coexisting tumors were located in different sections of the stomach, cases with collision tumors have also been reported [11]. In most cases, the coexisting epithelial tumor is a poorly differentiated intestinal type gastric adenocarcinoma, while atrophic gastritis, intestinal metaplasia or helicobacter pylori infection can also be present. 
Maiorana et al. [12] reported a series of 6 cases of synchronous occurrence of GISTs with gastric adenocarcinoma in 5 patients and one with carcinoid tumour. The coexisting stromal tumors are usually very small and are detected incidentally during surgery, as in our case.

Sanchez et al. [13] reported an incidence of $0.8 \%$ of incidentally finding small GISTs in patients who underwent laparoscopic Roux-en-Y gastric bypass. Various hypotheses have been made for the synchronous occurrence of a GIST with other tumors of different histogenesis. It has been suggested that gene mutations or a single carcinogenic agent might interact with adjacent tissues, inducing the development of different tumors of different histotypes in the same organ, while pure coincidence cannot be excluded. A multilineage progenitor cell has also been identified in murine stomach epithelium and it was postulated that it is the multilineage progenitor cell that actually accumulates the mutations, which eventually lead to the carcinogenesis $[14,15]$. GISTs that occur simultaneously with other neoplasms are usually small and of low or very low risk of malignancy and this probably reflects the fact that are discovered at a very early stage, before becoming symptomatic. In our case, they were limited in the muscularis propria and were circumscribed, well demarcated, and nodular.

Our case has much more in common with the case report of Salemis et al. [16], although the coexistent stromal tumor in their case was a single nodule. According to our knowledge, our finding of synchronous occurrence of adenocarcinoma with multiple small GISTs in the same organ, in what seemed like a sporadic case, is unique in the literature.

Benign GISTs outnumber malignant GISTs in the stomach. Tumors that are small (= or $<2 \mathrm{~cm}$ ) and show mitotic activity not exceeding 5 mitoses per 50 high-power fields have an excellent prognosis. However, a small proportion of tumors apparently lacking mitotic activity, do metastasize. Every GIST is now considered to be potentially malignant and small gastric GISTs less than $2 \mathrm{~cm}$ have a 100\% cure rate after complete surgical resection.

\section{Conclusion}

The coexisting GISTs are usually very small and are detected incidentally during surgery. In any case of GI neoplasm, the surgeon and the pathologist should be alert to perform a thorough preoperative and intraoperative investigation. Furthermore, incidental GISTs can be mistaken for a metastasis of the coexistent primary tumor, thus precluding its staging.

The most likely explanation for the presence of coexistent lesions may be just coincidence. Genetic factors have also been considered, although no information supporting such theories has been found. No significant family histories were reported in any patients. In our case also, the family history of the patient was unremarkable.

Our case report could provide further awareness and insight into the entity of concurrent tumors.

\section{Statement of Ethics}

The author has no ethical conflicts to disclose. Permission from the family of the diseased patient for the publication has been received.

\section{Disclosure Statement}

The author has no conflict of interest to declare. 


\section{References}

1 Casali PG, Blay JY; ESMO/CONTICANET/EUROBONET Consensus Panel of Experts. Gastrointestinal stromal tumours: ESMO Clinical Practice Guidelines for diagnosis, treatment and follow-up. Ann Oncol. 2010 May;21 Suppl 5:v98-102.

2 Wronski M, Ziarkiewicz-Wroblewska B, Gornicka B, Cebulski W, Slodkowski M, Wasiutynski A, et al. Synchronous occurrence of gastrointestinal stromal tumors and other primary gastrointestinal neoplasms. World J Gastroenterol. 2006 Sep;12(33):5360-2.

3 Kaffes A, Hughes L, Hollinshead J, Katelaris P. Synchronous primary adenocarcinoma, mucosa-associated lymphoid tissue lymphoma and a stromal tumor in a Helicobacter pylori-infected stomach. J Gastroenterol Hepatol. 2002 Sep;17(9):1033-6.

4 Usui M, Matsuda S, Suzuki H, Hirata K, Ogura Y, Shiraishi T. Somatostatinoma of the papilla of Vater with multiple gastrointestinal stromal tumors in a patient with von Recklinghausen's disease. J Gastroenterol. 2002;37(11):947-53.

5 Arnogiannaki N, Martzoukou I, Kountourakis P, Dimitriadis E, Papathanasaki A, Nastoulis E, et al. Synchronous presentation of GISTs and other primary neoplasms: a single center experience. In Vivo. 2010 Jan-Feb;24(1): 109-15.

6 Liszka Ł, Zielińska-Pajak E, Pajak J, Gołka D, Huszno J. Coexistence of gastrointestinal stromal tumors with other neoplasms. J Gastroenterol. 2007 Aug;42(8):641-9.

7 Agaimy A, Wünsch PH, Sobin LH, Lasota J, Miettinen M. Occurrence of other malignancies in patients with gastrointestinal stromal tumors. Semin Diagn Pathol. 2006 May;23(2):120-9.

8 Miettinen M, Lasota J. Gastrointestinal stromal tumors: review on morphology, molecular pathology, prognosis, and differential diagnosis. Arch Pathol Lab Med. 2006 Oct;130(10):1466-78.

9 Kang DY, Park CK, Choi JS, Jin SY, Kim HJ, Joo M, et al. Multiple gastrointestinal stromal tumors: clinicopathologic and genetic analysis of 12 patients. Am J Surg Pathol. 2007 Feb;31(2):224-32.

10 Lin YL, Tzeng JE, Wei CK, Lin CW. Small gastrointestinal stromal tumor concomitant with early gastric cancer: a case report. World J Gastroenterol. 2006 Feb;12(5):815-7.

11 Liu SW, Chen GH, Hsieh PP. Collision tumor of the stomach: a case report of mixed gastrointestinal stromal tumor and adenocarcinoma. J Clin Gastroenterol. 2002 Oct;35(4):332-4.

12 Maiorana A, Fante R, Maria Cesinaro A, Adriana Fano R. Synchronous occurrence of epithelial and stromal tumors in the stomach: a report of 6 cases. Arch Pathol Lab Med. 2000 May;124(5):682-6.

13 Sanchez BR, Morton JM, Curet MJ, Alami RS, Safadi BY. Incidental finding of gastrointestinal stromal tumors (GISTs) during laparoscopic gastric bypass. Obes Surg. 2005 Nov-Dec;15(10):1384-8.

14 Qiao XT, Ziel JW, McKimpson W, Madison BB, Todisco A, Merchant JL, et al. Prospective identification of a multilineage progenitor in murine stomach epithelium. Gastroenterology. 2007 Dec;133(6):1989-98.

15 Du S, Hasteh F, Weidner N, Shabaik A. A unique simultaneous presence of adenocarcinoma, carcinoid tumor and gastrointestinal stromal tumor (GIST) in the stomach. Internet J Pathol. 2009;8(2).

16 Salemis NS, Gourgiotis S, Tsiambas E, Karameris A, Tsohataridis E. Synchronous occurrence of advanced adenocarcinoma with a stromal tumor in the stomach: a case report. J Gastrointestin Liver Dis. 2008 Jun; $17(2): 213-5$. 